\title{
Identification of single nucleotide polymorphisms in the bovine interleukin-12 and interleukin-23 receptor genes and their associations with health and production traits in Holstein cows
}

\author{
A. Skelding, ${ }^{*}$ F. S. Schenkel, ${ }^{*}$ B. S. Sharma, ${ }^{*}$ C. Verschoor, ${ }^{*}$ S. Pant, ${ }^{*}$ G. Biggar, ${ }^{*}$ H. Boermans, $\dagger$ \\ and N. Karrow*1 \\ ${ }^{*}$ Department of Animal and Poultry Science, \\ †Department of Biomedical Science, University of Guelph, Guelph, Ontario, Canada N1G 2W1
}

\begin{abstract}
Interleukin-12 (IL-12) and interleukin-23 (IL-23) are proinflammatory cytokines produced by macrophages and dendritic cells in response to infection with intracellular pathogens. The IL-12 receptor (IL-12R) is a heterodimer composed of 2 subunits, $\beta 1$ and $\beta 2$. The IL-23 receptor (IL-23R) is a heterodimer composed of the IL-12R $\beta 1$ subunit and a unique IL-23R subunit. Given the importance of IL-12 and IL-23 for modulating inflammation and the host immune response, the IL-12 and IL-23 receptor genes may be suitable candidate genes for studying disease resistance in dairy cattle. We hypothesize that single nucleotide polymorphisms (SNP) exist within these genes and that they contribute to variation in health and production traits in dairy cattle. To investigate this, a selective DNA pool was constructed using bull semen based on the estimated breeding values for somatic cell score (SCS), an indicator trait used to achieve genetic improvement for resistance to mastitis. Gene segments were amplified from this pool by PCR and the amplicons were sequenced to reveal SNP. A total of 10 SNP, including 2 in IL-12R $\beta 1,5$ in IL-12R $\beta 2$, and 3 in IL-23R were identified. The SNP $(\mathrm{n}=5)$ were found in the $5^{\prime}$ untranslated region (UTR) putative promoter regions of the genes, and SNP IL-23R c.1714A > C was a nonsynonymous SNP. Canadian Holstein bulls $(\mathrm{n}=492)$ were genotyped using Sequenom MassARRAY (Sequenom Inc., San Diego, CA). No association was found with SCS based on bull deregressed estimated breeding values for SCS; however, associations of SNP in the IL-12R $\beta 2$ gene (c.-511A > G, c.87A > G, c.2957A > C) were found with milk and protein yield. Further
\end{abstract}

Received May 18, 2009.

Accepted June 9, 2010.

${ }^{1}$ Corresponding author: nkarrow@uoguelph.ca investigation will be required to elucidate the biological and practical relevance of these SNP.

Key words: interleukin-12, interleukin-23, somatic cell score, production trait

\section{INTRODUCTION}

Several genes involved in regulating inflammation and the immune response have been previously associated with health and production traits in dairy cattle. Genetic variants in the toll-like receptor-4 (TLR4; Sharma et al., 2006), caspase recruitment domain 15 (CARD15; Pant et al., 2007), and chemokine receptor 1 (CXCR1; Leyva-Baca et al., 2008) genes, for example, have been associated with SCS, a health trait that has been used for many years as an indicator trait to achieve genetic improvement for resistance to mastitis in dairy cattle (Shook and Schutz, 1994). Because of the strong correlation between SCS and clinical mastitis (Shook and Schutz, 1994), selection for decreased SCS has been demonstrated to be a successful approach for reducing clinical mastitis in dairy herds (Rupp and Boichard, 2003). In the context of production traits, variants in the genes encoding CARD15 (Pant et al., 2007), chemokine (C-C motif) ligand 2 (CCL2), IL-8, chemokine (C-C motif) receptor 2 (CCR2), and IL8RA (Leyva-Baca et al., 2007), and osteopontin (Alain et al., 2009) have been associated with milk and protein yields.

The identification of variants within genes that are associated with health and production traits can help improve accuracy of selection for these phenotypes when subsequently used in either marker-assisted or genome-wide selection. Additionally, it is imperative that we understand the relationship between SNP in immune-related genes and health and production traits in dairy cattle to ensure that selection of one trait does not negatively affect other priority traits.

The proinflammatory cytokines IL-12 and IL-23 are important regulators of inflammation and the host 
immune response. Interleukin-12 is a heterodimer composed of 2 covalently linked proteins, p35 and p40 (Yilmaz et al., 2005). It is generally produced by macrophages and dendritic cells in response to infection with intracellular bacteria (van de Vosse et al., 2003). Alluwaimi et al. (2003) investigated IL-12 expression in the Staphylococcus aureus-infected bovine mammary gland and found its transcriptional activity to be significantly increased at $24 \mathrm{~h}$ postinfection. Polymorphisms have been reported in the human IL-12 $\beta$ gene, which codes the p40 subunit of IL-12, and an A to C transversion in the $3^{\prime}$ untranslated region (UTR) of this gene was demonstrated to alter expression of IL-12 (Freidin et al., 2006). The IL-12 receptor (IL-12R) is composed of 2 subunits, $\beta-1$ and $\beta-2$, which are each coded by different genes. Interleukin-23 is a close relative of IL-12 and also plays an important role in the inflammatory response (Langrish et al., 2004). Interleukin-23 comprises a p19 subunit that associates with the IL-12p40 subunit (Langrish et al., 2004). Likewise, the IL-12R $\beta 1$ chain pairs with IL-23 receptor (IL-23R) subunit to form the high-affinity IL-23R.

Given the importance of these genes for regulating inflammatory and immune response and the potential that genetic variants within these genes may influence susceptibility of a variety of bovine inflammatory disorders, the objective of this study was to identify SNP in the bovine IL-12 and IL-23 receptor genes. Statistical analyses of these SNP were then undertaken to test for an association with dairy cattle health and production traits.

\section{MATERIALS AND METHODS}

\section{Bull Resource Population}

The resource population for this study consisted of 2,166 Holstein bulls, of which 492 were selected for genotyping based on their extreme EBV for SCS. Semen samples, donated by Semex Alliance (Guelph, Ontario, Canada), were used as a source of DNA. The selected bulls were from 25 half-sib sire families and the family sizes ranged from 15 to 30 bulls.

\section{Phenotypic Traits}

Estimated breeding values for all animals were provided by the Canadian Dairy Network (Guelph, Ontario, Canada). Deregressed EBV (drEBV) for SCS, 3 production traits, milk yield (MYLD), fat yield (FYLD), and protein yield (PYLD), and 2 healthrelated traits, udder depth (UDPTH) and lactation persistency (LACTP), were analyzed.

\section{DNA Extraction}

A standard phenol-chloroform procedure was used to extract DNA from semen samples, with a slight modification in centrifugation speed and time (Winfrey et al., 1997). An Eppendorf Biophotometer (Berlin, Germany) was used to assess the DNA concentration and quality based on absorbance of UV light at 260 and $280 \mathrm{~nm}$.

\section{SNP Discovery}

Twenty bulls with high EBV for SCS and 20 bulls with low EBV for SCS were selected to create a DNA pool containing equal amounts of DNA from each bull according to Leyva-Baca et al. (2008). The descriptive statistics of the bull EBV used for DNA pooling are shown in Table 1. This DNA pool was amplified via whole-genome amplification using the Qiagen REPLI-g ultra fast mini kit (Qiagen, Valencia, CA) and then used for SNP discovery in the bovine IL-12R $\beta 1$, IL$12 \mathrm{R} \beta 2$, and IL-23R genes. The bovine gene sequences for IL-12R $\beta 1$, IL-12R $\beta 2$, and IL-23R were accessed using Ensembl genome browser (http://www.ensembl. org/index.html). These sequences were used to design primers with the Primer3 software (http://biotools. umassmed.edu/bioapps/primer3_www.cgi) to amplify $1 \mathrm{~kb}$ of the putative promoter region, the $5^{\prime}$ UTR region, and the exons of each gene. The primer pairs are presented in Table 2. Amplification of DNA was performed via PCR with an Applied Biosystems GeneAmp PCR system-9700 thermocycler (Applied Biosystems, Foster City, CA). The PCR reactions were performed in a final volume of $10 \mu \mathrm{L}: 25 \mathrm{ng}$ of template DNA, 1 $\mu \mathrm{L}$ of each primer, $0.25 \mathrm{~m} M$ of each dNTP, $1.5 \mathrm{~m} M$ of $\mathrm{MgCl}_{2}, 1 \times$ PCR buffer $(200 \mathrm{~m} M$ Tris- $\mathrm{HCl}, \mathrm{pH}$ 8.4, 500 $\mathrm{m} M \mathrm{KCl}$,) and $0.75 \mathrm{U}$ of Taq polymerase. The PCR conditions were as follows: $94^{\circ} \mathrm{C}$ for $3 \mathrm{~min}, 35$ cycles of denaturation at $94^{\circ} \mathrm{C}$ for $30 \mathrm{~s}$, annealing temperature (depending on primer; see Table 2) for $30 \mathrm{~s}$, extension at $72^{\circ} \mathrm{C}$ for $30 \mathrm{~s}$, followed by a final extension at $72^{\circ} \mathrm{C}$ for $5 \mathrm{~min}$. Chromatograms for the forward and reverse DNA sequences were investigated to detect SNP in the candidate genes. In silico analysis of the $1 \mathrm{~kb}$ of the $5^{\prime}$ putative promoter region of each candidate gene was analyzed for potential transcription-factor binding sites using the software Genomatix (http://www.genomatix. de).

\section{SNP Genotyping}

The identified SNP ( $\mathrm{n}=10)$ were genotyped in 492 Holstein bulls using the Sequenom MassARRAY system (Sequenom Inc., San Diego, CA), which uses ma- 
Table 1. Descriptive statistics of the EBV of the Holstein bulls used for DNA pooling ${ }^{1}$

\begin{tabular}{|c|c|c|c|c|c|c|}
\hline \multirow[b]{2}{*}{ Group } & \multicolumn{6}{|c|}{ EBV } \\
\hline & $\begin{array}{l}\text { MYLD } \\
(\mathrm{kg})\end{array}$ & $\begin{array}{l}\text { FYLD } \\
(\mathrm{kg})\end{array}$ & $\begin{array}{l}\text { PYLD } \\
\text { (kg) }\end{array}$ & $\begin{array}{c}\text { UDPTH } \\
\text { (score) }\end{array}$ & $\begin{array}{c}\mathrm{SCS}^{2} \\
\text { (score) }\end{array}$ & $\begin{array}{r}\text { LACTP } \\
\text { (score) }\end{array}$ \\
\hline \multicolumn{7}{|l|}{ High group } \\
\hline Records (n) & 20 & 20 & 20 & 20 & 20 & 20 \\
\hline Mean & -142.33 & -7.07 & -1.24 & 3.84 & 3.47 & 100.51 \\
\hline $\mathrm{SD}$ & 794.38 & 34.52 & 25.75 & 3.26 & 0.30 & 4.74 \\
\hline Maximum & 1.304 .53 & 51.42 & 37.91 & 10.73 & 4.02 & 109.09 \\
\hline Minimum & $-1,374.16$ & -74.12 & -50.08 & -1.01 & 3.06 & 92.07 \\
\hline \multicolumn{7}{|l|}{ Low group } \\
\hline Records (n) & 20 & 20 & 20 & 20 & 20 & 20 \\
\hline Mean & -207.34 & -6.95 & -7.61 & 4.80 & 2.59 & 102.18 \\
\hline $\mathrm{SD}$ & 902.53 & 33.55 & 23.69 & 4.18 & 0.21 & 5.62 \\
\hline Maximum & $1,443.76$ & 58.82 & 41.61 & 13.23 & 2.99 & 112.60 \\
\hline Minimum & $-2,452.47$ & -64.36 & -66.00 & -1.41 & 2.30 & 88.42 \\
\hline
\end{tabular}

${ }^{1}$ MYLD $=$ milk yield; FYLD $=$ fat yield PYLD = protein yield; UDPTH = udder depth; LACTP = lactation persistency. One bull had missing EBV for udder depth and SCS.

${ }^{2} \mathrm{SCS}[\log (2)$ scores] was used for selecting the 20 high- and 20 low-EBV bulls for DNA pooling.

trix-assisted laser desorption/ionization time-of-flight (MALDI-TOF) mass spectrometry to analyze SNP in amplified DNA fragments (Tost and Gut, 2005).

\section{Statistical Analyses}

Hardy-Weinberg Equilibrium and Linkage Disequilibrium Analysis. The genotype frequencies were tested for deviations from proportions of HardyWeinberg equilibrium, by chi-squared test. The pairwise level of linkage disequilibrium was measured using the squared correlation $\left(\mathrm{r}^{2}\right)$ of the alleles at 2 loci (Hill and Robertson, 1968).

Allele Substitution Effect. Associations of the SNP with traits of interest for each breed were evaluated by fitting a single-trait mixed inheritance model (SNP allele substitution effects plus polygenic effects). The statistical model was

$$
Y_{k}=\mu+\sum_{j=1}^{n} \beta_{j} G_{e n}+P_{j o l}+e_{k}, k=\{1, \ldots, 492\}
$$

where $Y_{k}$ is the trait drEBV for the $k$ th bull; $\mu$ is the overall mean for the trait drEBV; $n$ is the number of SNP (i.e., 2, 4, and 4 in case of IL12R $\beta 1$, IL12R $\beta 2$, and IL23R, respectively); $\beta_{j}$ is the fixed linear regression coefficient (average allele substitution effect) for the $j$ th SNP and $G_{e n}$ is the recoded genotype of the $k$ th bull for the $j$ th SNP; Pol $_{k}$ is the random additive genetic (polygenic) effect of the $k$ th bull; and $e_{k}$ is the residual random effect associated with the $k$ th bull. The polygenic and residual effects were assumed distributed as $N\left(0 ; \mathbf{A} \sigma_{a}^{2}\right)$ and $N\left(0 ; \mathbf{E} \sigma_{e}^{2}\right)$, respectively, where $\sigma_{a}^{2}$ and $\sigma_{e}^{2}$ are the additive genetic and residual variances, respec- tively; $\mathbf{A}$ is the numerator additive relationship matrix (Henderson, 1988) that was based on the available pedigree information (987 animals), and $\mathbf{E}$ is a diagonal matrix with diagonal elements equal to the inverse of the effective number of daughters of each bull.

The SNP genotypes were recoded as in Zeng et al. (2005):

$$
G e n_{j k}=\left\{\begin{array}{l}
1 \text { for a homozygote } 1 \\
0 \text { for a heterozygote } \\
-1 \text { for a homozygote } 2
\end{array}\right.
$$

Haplotype Analyses. The haplotype probabilities were reconstructed using the HAPROB program developed by Boettcher et al. (2004). This program uses a 2-step Monte Carlo-based algorithm to estimate haplotype probabilities for the genotyped members of half-sib families where the parents lack genotypic information. Haplotype effects were estimated by regressing drEBV on haplotype probabilities. The following single-trait model was used for statistical analysis:

$$
Y_{k}=\mu+\sum_{j=1}^{h} \beta_{j} H_{a p}+\operatorname{Pol}_{k}+e_{k}
$$

where $Y_{k}$ is the trait drEBV for the $k$ th bull; $\mu$ is the overall mean for the trait drEBV; $\beta_{j}$ is the linear regression coefficient for the $j$ th haplotype; $H_{a p}$ is the probability of the $j$ th haplotype for the $k$ th bull and $h$ is the number of haplotypes fit in the analysis (i.e., 4, 10, and 7 in case of IL12R $\beta 1$, IL12R $\beta 2$, and IL23R, respectively); $\mathrm{Pol}_{k}$ is the random additive genetic (polygenic) effect of the $k$ th bull; and $e_{k}$ is the random residual effect 
Table 2. Primers for identification of SNP in IL-12 receptor subunit $\beta 1$ (IL12R $\beta 1$ ), IL-12 receptor subunit $\beta 2$ (IL12R $\beta 2$ ), and IL-23 receptor (IL23R) genes

\begin{tabular}{|c|c|c|c|}
\hline Gene fragment & Direction & Primer sequence, $5^{\prime}-3^{\prime}$ & $\mathrm{T}_{\mathrm{ann}}{ }^{1}\left({ }^{\circ} \mathrm{C}\right)$ \\
\hline \multicolumn{4}{|c|}{$\mathrm{IL}_{12 \mathrm{R}} \beta 15^{\prime} \mathrm{UTR}^{2}$} \\
\hline \multirow{2}{*}{ Part $1(-221)$} & Forward & GGCTGCCATTGCCTTCTC & 61 \\
\hline & Reverse & GTCCTTGAAGAGGGCAGCAG & 60 \\
\hline \multirow[t]{2}{*}{ Part $2(-521)$} & Forward & AGGAAACAAGGAAATTCTTCAGACA & 61 \\
\hline & Reverse & CTGAGCCCTAGACCCTGAGCTATAC & 62 \\
\hline \multirow[t]{2}{*}{ Part $3(-840)$} & Forward & CTGGCAATCATTCTACCTTCTGTCT & 62 \\
\hline & Reverse & CTCTCTGGCCCACTTCTGAGAT & 62 \\
\hline \multicolumn{4}{|l|}{ IL12R $\beta 1$ gene } \\
\hline \multirow[t]{2}{*}{ Exon 1} & Forward & GGGCCGTGTCTCATAACTTTTT & 62 \\
\hline & Reverse & AACTAGCCCTCCACATACACACAAC & 63 \\
\hline \multirow[t]{2}{*}{ Exon 2} & Forward & ATAGGTGGGAAAGTGGATAGGTGT & 61 \\
\hline & Reverse & TGCTAGGGAGGAGTCAGTCAGAG & 62 \\
\hline \multirow[t]{2}{*}{ Exon 3} & Forward & TCCCTGAGTTGAGTCAGTGTGG & 63 \\
\hline & Reverse & CAGGAAGGGGCACAGACC & 62 \\
\hline \multirow[t]{2}{*}{ Exon 4} & Forward & GAAAGGACAGTGGGTTGTGATG & 62 \\
\hline & Reverse & ATGGGAAAACAGCTGGCACTAT & 62 \\
\hline \multirow[t]{2}{*}{ Exon 5} & Forward & GATTTTCAGGACCAGCAACTCAC & 62 \\
\hline & Reverse & GGGGTGATAGATACAGAGACTGCAA & 63 \\
\hline \multirow[t]{2}{*}{ Exon 6} & Forward & TGTGTGGCATTTAGCAAAATAATTG & 62 \\
\hline & Reverse & AGTCAGACACAACTGAAGCGACTTA & 62 \\
\hline \multirow{2}{*}{ Exon 7} & Forward & GCTTCATATGTACATGGTGGCTAAG & 61 \\
\hline & Reverse & CACCTTATTTCTGATCCCACTACCA & 63 \\
\hline \multirow{2}{*}{ Exon 8} & Forward & AAGGTCCCATGTCTTCATCCAG & 63 \\
\hline & Reverse & TTACACATTTCCACTCTAGCCTCCT & 62 \\
\hline \multirow[t]{2}{*}{ Exon 9} & Forward & GAGTCTGTGTTCATGGGCTGAAT & 63 \\
\hline & Reverse & CCTAGTCTAGCTGAGGTTCAGATGC & 62 \\
\hline Exon 10 & Forward & GGGGAGACAGAGTCAGATACAAACA & 63 \\
\hline & Reverse & ATGGAGGATGCCTATAGGTTTGAGT & 62 \\
\hline Exon 11 & Forward & TGCTCTAAAAGTTCCTGGTAGATGG & 62 \\
\hline & Reverse & GGTATTCCCCATTCCTTATATCCAA & 62 \\
\hline Exon 12 & Forward & GAGGCGGAACATGCTGAG & 61 \\
\hline & Reverse & CACATAGCAAGGCAGACTCCAG & 62 \\
\hline Exon 13 & Forward & GTTTTCCACAAAATGTCTGTGAGC & 62 \\
\hline & Reverse & GTCCATGGGACTGCAAAGAGTT & 63 \\
\hline Exon 14 & Forward & CAGGGTCAGCAGTGAGCTGAT & 63 \\
\hline & Reverse & GGACATAAAAACCTATTTGGGGCTA & 62 \\
\hline Exon 15 & Forward & TCCTTATTGTAACCTAATGGAGATGAA & 60 \\
\hline & Reverse & CTACTCTTCAGAGCCCTAATCCAGA & 62 \\
\hline Exon 16 & Forward & GGATTTTAGAGAATTTAGCCGGAAG & 62 \\
\hline & Reverse & GGATCAGCAGTGGGGACCTA & 62 \\
\hline IL12R $\beta 2$ promo & & & \\
\hline Part 1 & Forward & AAGAAAATGTCTTGAGTGCTGAGGTC & 63 \\
\hline & Reverse & TGCAAGTTGGTGATTTCTGTTTGTT & 63 \\
\hline Part 2 & Forward & GAATTTCTGGCCCACTTTTGATAGA & 64 \\
\hline & Reverse & AGTCTCACCCAAAGCTTTTTCCTTC & 64 \\
\hline Part 3 & Forward & TTCTCACTGCTCTTGGAATTTTAACAC & 63 \\
\hline & Reverse & ATTCAGAGGGCTTAAAATGCTCCTT & 63 \\
\hline IL12R $\beta 2$ gene & & & \\
\hline Exon 1 & Forward & AAGGAGCATTTTAAGCCCTCTGAAT & 64 \\
\hline & Reverse & CCACTTAAACTTGACCGATTGCTTT & 64 \\
\hline Exon 2 & Forward & TAGGGTTTGTTTTATCCAGCTTTGG & 63 \\
\hline & Reverse & TCCTTGAAGTGGGAGAAGAAAGAAC & 63 \\
\hline Exon 3 & Forward & GATGGGAGACATAGGGGAAGTATTGT & 64 \\
\hline & Reverse & CTGGAGGTGTTTACTGCTTCTCCTC & 64 \\
\hline Exon 4 & Forward & TGTTTCTTTGTGCTAAAAAGAGGCAAT & 64 \\
\hline & Reverse & CGTTTGAGACCTACATCCTTTTGCT & 64 \\
\hline Exon 5 & Forward & TTCTAAAAGCCAGGGACTCCTCTCT & 64 \\
\hline & Reverse & ATCTGGGCGGTGCTACTAAGAATTT & 64 \\
\hline Exon 6 & Forward & TTGAATGACAGATTTGAAAAGTAAAAAGG & 63 \\
\hline & Reverse & TCTTTCATCCTGTCTATCCCCACTC & 63 \\
\hline Exon 7 & Forward & AAAGGAAAAGGGAAATACTGGGACA & 64 \\
\hline & Reverse & AAGTTCTTCGGCCTTGAAACTTACC & 64 \\
\hline Exon 8 & Forward & TAGTCACCTGCTTCAGGCAGTCAT & 64 \\
\hline & Reverse & TGGGCCTGAATGAGCTAAGAGATTA & 64 \\
\hline Exon 9 & Forward & AACTTTGCTGGGTTGGGTAGTTGT & 64 \\
\hline
\end{tabular}


Table 2 (Continued). Primers for identification of SNP in IL-12 receptor subunit $\beta 1$ (IL12R $\beta 1$ ), IL-12 receptor subunit $\beta 2$ (IL12R $\beta 2$ ), and IL-23 receptor (IL23R) genes

\begin{tabular}{|c|c|c|c|}
\hline Gene fragment & Direction & Primer sequence, $5^{\prime}-3^{\prime}$ & $\mathrm{T}_{\text {ann }}{ }^{1}\left({ }^{\circ} \mathrm{C}\right)$ \\
\hline & Reverse & TCTCACACAGCTGTAACTGAGAGCA & 64 \\
\hline \multirow[t]{2}{*}{ Exon $10-11$} & Forward & CAGAAGCTGTCTGTGTGGTTTTGTT & 64 \\
\hline & Reverse & AGCTTGTGTTTTCCCAACACCTAAA & 64 \\
\hline \multirow[t]{2}{*}{ Exon 12} & Forward & ATGGGGCCTTCATAAATGATAGTGT & 63 \\
\hline & Reverse & GGGTATACTCCTATGGAGGAATCCAG & 64 \\
\hline \multirow[t]{2}{*}{ Exon 13} & Forward & ACTCTCCAGGCAAGAACACTGGA & 64 \\
\hline & Reverse & CCCATAGCAGCACCAGCTATTATATG & 64 \\
\hline \multirow[t]{2}{*}{ Exon 14} & Forward & GCATAATCCTGCACTGAAATCACCT & 64 \\
\hline & Reverse & ATGGGAGACTTAGAACCCCATCAAC & 64 \\
\hline \multirow{2}{*}{ Exon $15-1$} & Forward & ACAGTGTTCTGCCTTGAGCTGACTA & 64 \\
\hline & Reverse & TGTATAGATCCACCCCTTGTCCTGT & 64 \\
\hline \multirow[t]{2}{*}{ Exon $15-2$} & Forward & CAAGCCCACCACCTCCAAG & 64 \\
\hline & Reverse & GAGATTCACCCTGAGCTGGCTTAC & 64 \\
\hline \multirow[t]{2}{*}{ Exon $15-3$} & Forward & AGTGGTCATTACCTGTGGGTGCT & 64 \\
\hline & Reverse & ACACGACTGAGCGACTGATCTGA & 64 \\
\hline \multicolumn{4}{|c|}{ 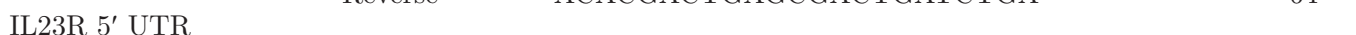 } \\
\hline \multirow[t]{2}{*}{ Part $1(-123)$} & Forward & CAAGGGTCAAAATGTCTTATGAAGG & 62 \\
\hline & Reverse & TCCATGACACCAACTGAAGAATATG & 62 \\
\hline \multirow[t]{2}{*}{ Part $2(-540)$} & Forward & AATGGCAATATTGTTTGGAATTTCT & 61 \\
\hline & Reverse & GAAGAAAGGAACAAAGGGAGGAAG & 62 \\
\hline \multirow[t]{2}{*}{ Part $3(-763)$} & Forward & TCCTAACTAGGAATATTTCACAGTTTCTTT & 60 \\
\hline & Reverse & CCAAAAAGATCAGAACTTCCAGAAA & 62 \\
\hline \multirow[t]{2}{*}{ Part $4(-883)$} & Forward & CATACAGAGTTATGATAGAAGCTGAATCTG & 61 \\
\hline & Reverse & TTGCCATTAGTTTACATCTGGAGTT & 60 \\
\hline \multicolumn{4}{|c|}{ 19 } \\
\hline \multirow[t]{2}{*}{ Exon 1} & Forward & TCСТTCСТCССТTTGTTCCTT & 61 \\
\hline & Reverse & GGAGGGAGGTTGGACTCACA & 62 \\
\hline \multirow[t]{2}{*}{ Exon 2} & Forward & GCAAAGCATATTTTTCTGAATATCTGG & 62 \\
\hline & Reverse & CATTAATTTGCCTCCATTTTTAGACA & 62 \\
\hline \multirow[t]{2}{*}{ Exon 3} & Forward & TTCCAAATGAAATGGAATGGTTATC & 62 \\
\hline & Reverse & ACAGCACTGGCTGAACTGGAG & 63 \\
\hline \multirow[t]{2}{*}{ Exon 4} & Forward & CCTGGATGGTCTTGATTATGTTTTC & 62 \\
\hline & Reverse & TTTGATTAGGTCAGCGCAAATTAGT & 62 \\
\hline \multirow[t]{2}{*}{ Exon 5} & Forward & TTGTTTAGCACTTTGAGTTTTAACAGG & 61 \\
\hline & Reverse & AGTTTGAATATCACACAAACAGTCAGA & 60 \\
\hline \multirow[t]{2}{*}{ Exon 6} & Forward & TGCCCCATATGTTTCTGCTAATAAT & 62 \\
\hline & Reverse & TGAGCGACTGAACTTAACTGACTTG & 62 \\
\hline \multirow[t]{2}{*}{ Exon 7} & Forward & AAACTCTCTTAGGAAACTGGGCAAC & 62 \\
\hline & Reverse & CGTGATTTTTCAGTATGGAGCATTT & 63 \\
\hline \multirow[t]{2}{*}{ Exon 8} & Forward & GAGTCAGTTCTTCACATCACGTAGC & 62 \\
\hline & Reverse & СCTTTTATTTTGCCTTTTTCTCAGG & 62 \\
\hline \multirow[t]{2}{*}{ Exon 9} & Forward & ATTTCCTAATCTCCAAGATGATTGC & 61 \\
\hline & Reverse & AACTATTGGACTGTGCTCTTGGTCT & 62 \\
\hline \multirow[t]{2}{*}{ Exon $10-1(+350)$} & Forward & AGTCGAGGGAAAGCAAAATTCTAGT & 62 \\
\hline & Reverse & GAATTTGTACTTGAGACAGAAAAAGCA & 61 \\
\hline Exon $10-2(+651)$ & Forward & CATCTCAGCAATGATGATGAAACA & 62 \\
\hline & Reverse & ATTGGGCAGAATTAAATTTGTGTTG & 62 \\
\hline
\end{tabular}

${ }^{1} \mathrm{~T}_{\mathrm{ann}}=$ annealing temperature.

${ }^{2} \mathrm{UTR}=$ untranslated region

associated with the $k$ th bull. As for the allele substitution effect analysis, the polygenic and residual effects were assumed distributed as $N\left(0 ; \mathbf{A} \sigma_{a}^{2}\right)$ and $N\left(0 ; \mathbf{E} \sigma_{e}^{2}\right)$, as defined previously. Both the allele substitution effect and the haplotype effect analyses were carried out using the ASREML software (Gilmour et al., 2000).

\section{RESULTS}

\section{SNP Discovery}

Investigation of exonic and promoter sequences of IL-12R $\beta 1$, IL-12R $\beta 2$, and IL-23R revealed the presence of 10 SNP (Figure 1). Five SNP were found in the 5 upstream region in the putative promoter region of the IL-12R $\beta 2$ and IL-23R genes (IL-12R $\beta 2$ c.-756T > C, IL- $12 R \beta 2$ c. $-511 \mathrm{~A}>\mathrm{G}$, IL- $12 \mathrm{R} \beta 2$ c.-406A $>$ G, IL$23 \mathrm{R}$ c. $-856 \mathrm{~A}>\mathrm{G}$, and IL-23R c. $-207 \mathrm{~T}>\mathrm{C}), 4 \mathrm{SNP}$ were found within the coding regions of the IL-12R $\beta 1$, IL-12R $\beta 2$, and IL-23R genes (IL- $12 R \beta 1$ c. $81 \mathrm{~T}>\mathrm{C}$, IL$12 \mathrm{R} \beta 1$ c. $1500 \mathrm{~T}>\mathrm{C}$, IL-12R $\beta 2$ c. $87 \mathrm{~A}>\mathrm{G}$, and IL-23R c. $1714 \mathrm{C}>\mathrm{A}$ ), and $1 \mathrm{SNP}$ was found in the $3^{\prime}$ UTR of IL-12R $\beta 2$ (IL-12R $\beta 2$ c.2957A > C). The SNP IL-12R $\beta 1$ c. $81 \mathrm{~T}>\mathrm{C}$ was found in exon 2 and SNP IL-12R $\beta 1$ c.1500T > C was found in exon 13; both SNP are syn- 
A.

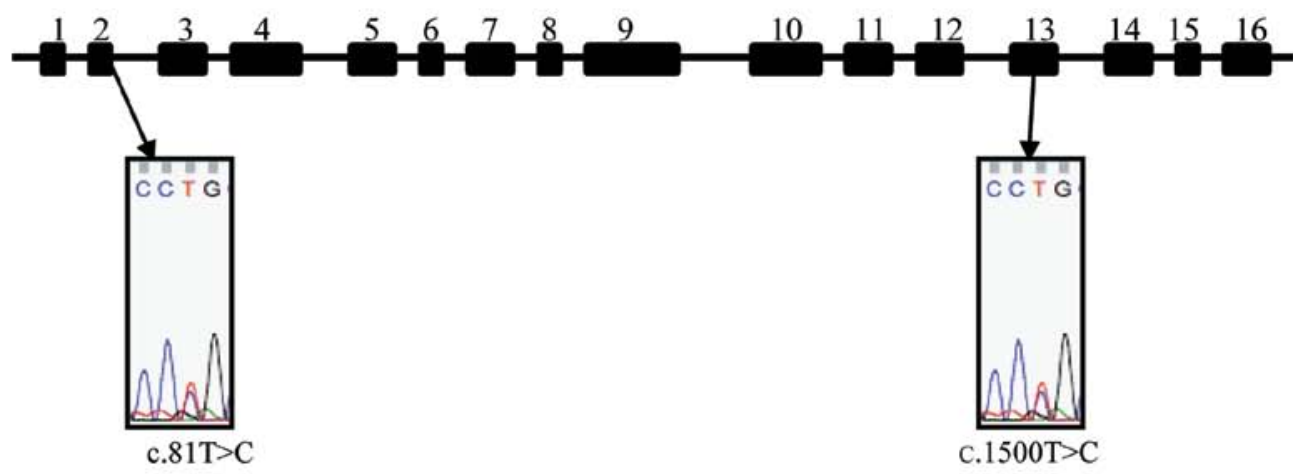

B.
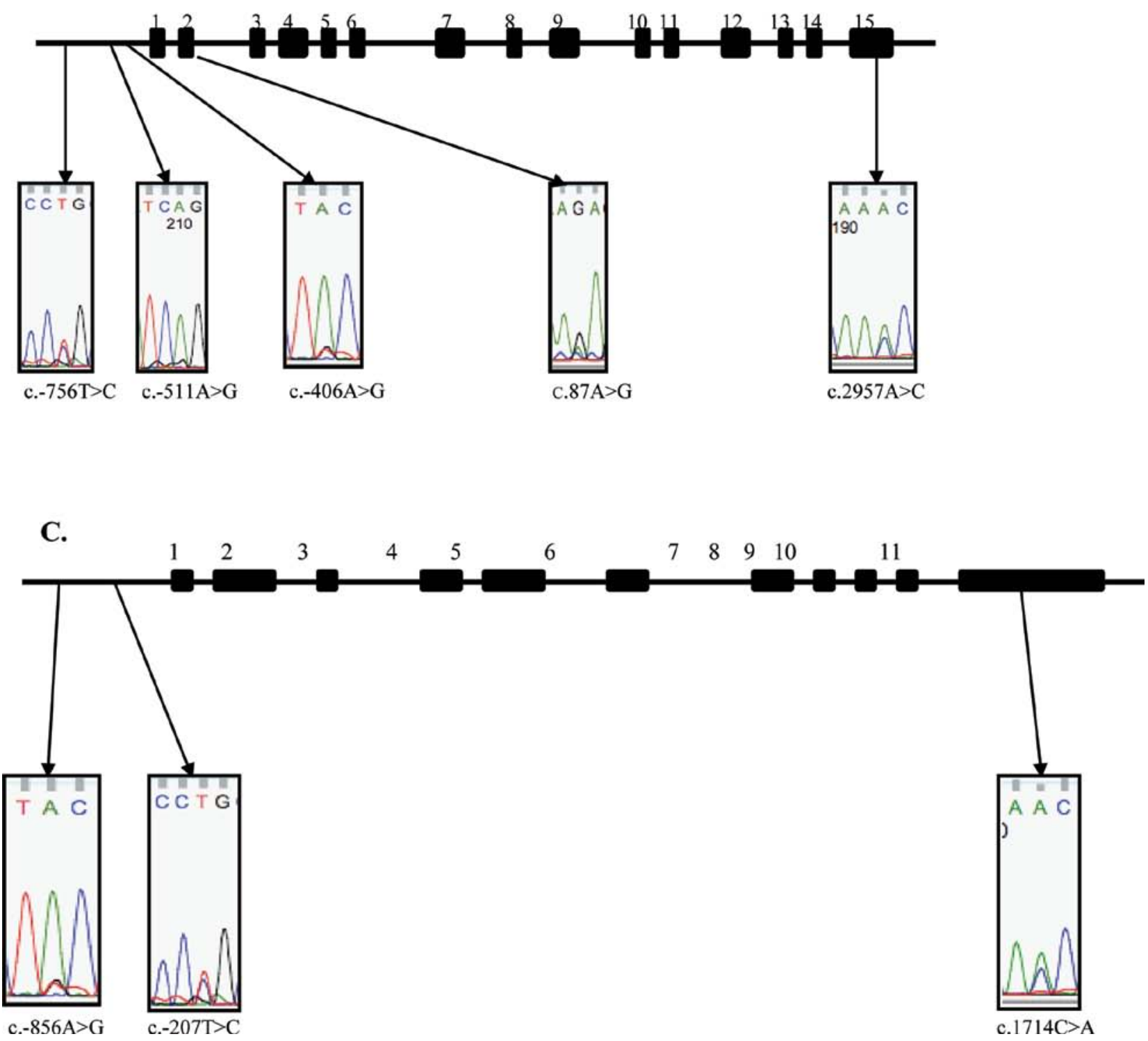

Figure 1. Position of SNP identified in the IL-12 receptor subunit $\beta 1$ (IL12R $\beta 1$ ) gene (A), IL-12 receptor subunit $\beta 2$ (IL12R $\beta 2$ ) gene (B), and the IL-23 receptor (IL23R) gene (C). Color version available in the online PDF. 
TTGAAAAGATGCTAAATACAGGAGTCAAAAAAGCTAGGGGATAGTTTCCAGG AAGCCAGCTAGAGCAGGACTGTAGTATCTCTAGCACTGAACACCGCACCTGG CAGATGAAAGGTAACCATCACTGTTGAATGAATAAGTGAACTTTTCTGAGGC

\section{Oct}

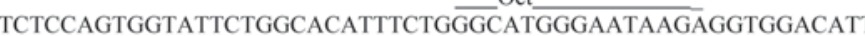
GGCTGGTTGCCAACAGCCCAACATCACACAGCTTTTCAGCCT/CGCTTCTTGA ATTTCTGGCCCACTTTTGATAGAATTTAGAGAATTTGCTAGAGCCATTCCTTA

NF-AT

GGTTTGTATTTGGAAACAAACAGAAATCACCAACTTGCATTTTCCAAATCATT

GATA

TAAGATAAAAATATTCAGTAAACTAAATAAATAATGAATAAACAAGCAAAC

AAGGAAAAGTGAACCTATGGAAAACCCAAACAGCCCCCTGGGAACCCTTGG CLOX

$\overbrace{\text { STAT6 }}^{{ }_{\text {DEAF }}{ }_{\text {NR2F }}{ }^{\mathrm{CLOX}} \_}$

AATTCTTTTCTTACTGCCAAGGAAATCA/GGTCTTTTACTGAGTTGTGGAGTGT GCGGGGTAGTAGTGGTTATGAACCACTTTTTAATCTCCTGTTACTTCCAGGAG $\mathrm{NR} 2 \mathrm{~F}$ $\mathrm{POZ}$

CACATGACTAGATCAGAAATTCTCACTA/GCTCTTGGAATTTTAACACTAGTA CATTTGACTATGATGACGCAGACTTGTTTGGAAGGAAAAAGCTTTGGGTGAG

ACTGAATCTCAGATTAAGTAACTTGCCCATAGGCTCAAAGTATTTAAACCCA

NR2F

GATA

GTTCTAAAGGACTCCATAGGTCAGATCTCTTCGCTTACTCTTTGCTGACCCCA AGAAGCTTAAATGACATGAGGGTTAGAGGTGGCTAAGATTTTTAGTTTATTG GTTTTAAACATCATTTTTTAGAAAGTCTCTTAACAACACAGAGTAAATTAAAG GAGCATTTTAAGCCCTCTGAATTGTTTTGGTTGTGGTATTTTCTGAATGTATTT

NF-AT

AACATGAATGAATTTGTCTTCTTTTACAAGGAAACATACAGAGGTGTATTCCA GAATTGACTGTTC +1

Figure 2. Bovine IL-12 receptor subunit $\beta 2$ (IL12R $\beta 2$ ) 5'-flanking region sequence $(-700 \mathrm{bp})$, and putative binding sites for bovine transcription factors (overlined). The start codon is localized at +1 . Transition SNP c.-756 (T/C), c.-511 (A/G), and c.-406 (A/G) are shown in bold font. Oct $=$ octamer binding protein; NF-AT $=$ nuclear factor of activated $\mathrm{T}$ cells; GATA = GATA-binding factor; CLOX $=$ CLOX homology factors; DEAF $=$ epidermal autoregulatory factor; $\mathrm{NR} 2 \mathrm{~F}=$ nuclear receptor subfamily 2 factors; STAT6 $=$ signal transducer and activator of transcription $6 ; \mathrm{POZ}=\mathrm{POZ}$ domain zinc finger.

onymous. The SNP IL-12R $\beta 2$ c.87A > G, found in exon 2 , is also synonymous. Single nucleotide polymorphism IL-23R c.1714C > A, found in exon 11, is nonsynonymous, with the A allele coding for threonine and the $\mathrm{C}$ allele coding for proline. All the SNP were submitted to the National Center for Biotechnology Information (http://www.ncbi.nlm.nih.gov/) and were assigned the following reference numbers: RefSNP IL-12R $\beta 1$ c.81T $>\mathrm{C}$ ss104807459, IL-12R $\beta 1$ c. $1500 \mathrm{~T}$ > C ss104807460, IL-12Rß2 c.-756T > C ss104807454, IL-12Rß2 c.-511A $>$ G ss104807455, IL-12R $\beta 2$ c.-406A > G ss104807456, IL-12Rß2 c.87A > G ss104807457, IL-12Rß2 c.2957A $>\mathrm{C}$ ss104807458, IL-23R c.-856A > G ss104807461, IL-23R c.-207T > C ss104807462, IL-23R c.1714C > A ss104807463.

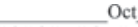

TCTGTATGATATTTCATACAGAGTTATGATAGAAGCTGAATCTGTAATATTATCTAACATCATA TAT $\overline{\text { GATA }}$ TATA TCGCTTTAAATGATATTATTGTGTATACTCTATTAGGTGAGTAGTTAACTCCTAACTAGGAATA CLOX

TTTCACAGTTCTTTTA/GTCTATTATTTACAAAGAGAAGATCTACTATTATTAACAAATCATGA TATA

AAGGGATCTTTATATTCATAAGTCACTGGGGACCTCAGTTTTTAAAAATGAAATATTGTGTGC CATTTGATGAGTTATGAGTTTTTGTTGTTTTATATGAAAGGCTCAGGTTTAATTAAATAACTGA CATTTGATGAGTTATGAGTTTTTGTTGTTTTATATGAAAGGCTCAGGTTTAATTAAATAACTGA

— TATA__ STAT6

TAACTCCAGATGTAAACTAATGGCAATATTGTTTGGAATTTCTATAATGTGCTTCTTTTGAACT GATA GATA

СTCTGCAGATAAAGGGGATTTCTGTAAATCTAACTGGTGATA $\overline{\text { TATGTG }} \overline{\text { TAAGTTTTGTCTCAC }}$

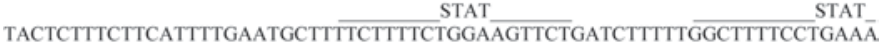

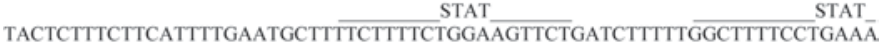

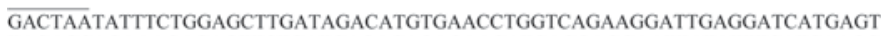
TAGAGCAGAAAGAGAGTTCTGATGAGAGAGACAAGATCAGGAGGAATTATATTTCATCAAGG TATA

GTCAAAATGTCTTATGAAGGTAATTCACACTGATTTTTGAAGGGTATAAAATCCCATTCTTTTO PAX

CTTTACTCCTCCACCTTATTATACCTCCT/CCACACCCCAGTGACTCTTCTTTGCCCTGTTTCCTT CTCССТTCCСATCCTTCCTCCCTTTGTTCCTTTCTTCCTAATTAAAGTTTCTCATCAAAAACAAC NF-AT

TGCTAGGAAAAATGTTATGCC TTTTAGTATTTTATTAAAATATTACAGTTTAAACATTTTTCAT ATTTTTCTAGAGAGCAACAAAGAGTTTTTTCTGCTTTCAGGC+1

Figure 3. Bovine IL-23 receptor (IL23R) 5'-flanking region sequence $(-1,000 \mathrm{bp})$, and putative binding sites for bovine transcription factors (overlined). The start codon is localized at +1 . Transition SNP c.-856(A/G) and c.-207 (T/C) are shown in bold font. Oct = octamer binding protein; TATA = TATA-binding protein factor; GATA $=$ GATA-binding factor; CLOX $=$ CLOX homology factor; STAT $=$ signal transducer and activator of transcription; PAX $=$ PAX paired domain binding site; NF-AT $=$ nuclear factor of activated $\mathrm{T}$ cells.

In silico analysis of the $5^{\prime}$ upstream region revealed that SNP IL-12R $\beta 2$ c.-511A > G is positioned within 4 potential transcription factor binding sites, the transcription factors being STAT6, NR2F, CLOX, and DEAF1 (Figure 2). The SNP IL-12Rß2 c.-406A > G is positioned within the potential transcription factor POZ binding site (Figure 2). The SNP IL-23R c.-856A $>\mathrm{G}$ is positioned within 9 potential transcription factor binding sites, the transcription factors being ISRE, MEF2, EVI1, CLOX, GATA1, BRN3, SATB1, HNF1, and CUT2 (Figure 3). Finally, SNP IL-23R c.-207T > $\mathrm{C}$ is positioned within 3 potential transcription factor binding sites, the transcription factors being BRACH, PAX6, TIEG (Figure 3).

\section{Statistical Analyses}

Hardy-Weinberg Equilibrium and Linkage Disequilibrium Analyses. The observed genotypic and allelic frequencies are summarized in Table 3. The individual frequencies of the genotypes were in HardyWeinberg equilibrium for all but 2 of the SNP (IL-12R 32 
Table 3. Genotypic and allelic frequencies of SNP found in IL-12 receptor subunit $\beta 1$ (IL12Rß1), IL-12 receptor subunit $\beta 2$ (IL12R $\beta 2$ ), and IL-23 receptor (IL23R) genes in Holstein cattle

\begin{tabular}{|c|c|c|c|c|}
\hline \multirow[b]{2}{*}{ SNP } & \multicolumn{2}{|c|}{ Genotype } & \multicolumn{2}{|c|}{ Allele } \\
\hline & $\mathrm{n}$ & Frequency (\%) & $\mathrm{n}$ & Frequency (\%) \\
\hline \multicolumn{5}{|l|}{ IL12R $\beta 1$} \\
\hline \multicolumn{5}{|l|}{ c. $81 \mathrm{~T}>\mathrm{C}$} \\
\hline $\mathrm{TT}(\mathrm{T})$ & 181 & 36.6 & 599 & 60.5 \\
\hline $\mathrm{TC}$ & 237 & 47.9 & & \\
\hline CC (C) & 77 & 15.6 & 391 & 39.5 \\
\hline \multicolumn{5}{|c|}{ c. $1500 \mathrm{~T}>\mathrm{C}$} \\
\hline $\mathrm{TT}(\mathrm{T})$ & 258 & 51.6 & 710 & 71.0 \\
\hline $\mathrm{TC}$ & 194 & 38.8 & & \\
\hline $\mathrm{CC}(\mathrm{C})$ & 48 & 9.6 & 290 & 29.0 \\
\hline \multicolumn{5}{|c|}{ IL12R $\beta 2$} \\
\hline \multicolumn{5}{|c|}{ c. $-756 \mathrm{~T}>\mathrm{C}$} \\
\hline TT (T) & 206 & 41.5 & 660 & 66.5 \\
\hline $\mathrm{TC}$ & 248 & 50.0 & & \\
\hline $\mathrm{CC}(\mathrm{C})$ & 42 & 8.5 & 332 & 33.5 \\
\hline \multicolumn{5}{|c|}{ c. $-511 \mathrm{~A}>\mathrm{G}$} \\
\hline $\mathrm{AA}(\mathrm{A})$ & 425 & 85.7 & 921 & 92.8 \\
\hline $\mathrm{AG}$ & 71 & 14.3 & & \\
\hline $\mathrm{GG}(\mathrm{G})$ & 0 & 0 & 71 & 7.2 \\
\hline \multicolumn{5}{|c|}{ c. $-406 \mathrm{~A}>\mathrm{G}$} \\
\hline AA (A) & 425 & 85.7 & 921 & 92.8 \\
\hline $\mathrm{AG}$ & 71 & 14.3 & & \\
\hline GG (G) & 0 & 0 & 71 & 7.2 \\
\hline \multicolumn{5}{|l|}{ c. $87 \mathrm{~A}>\mathrm{G}$} \\
\hline $\mathrm{AA}(\mathrm{A})$ & 18 & 3.7 & 241 & 24.3 \\
\hline $\mathrm{AG}$ & 205 & 41.3 & & \\
\hline GG (G) & 273 & 55.0 & 751 & 75.7 \\
\hline \multicolumn{5}{|c|}{ c. $2957 \mathrm{~A}>\mathrm{C}$} \\
\hline $\mathrm{AA}(\mathrm{A})$ & 219 & 44.1 & 669 & 67.4 \\
\hline $\mathrm{AC}$ & 231 & 46.6 & & \\
\hline CC (C) & 46 & 9.3 & 323 & 32.6 \\
\hline \multicolumn{5}{|c|}{ IL23R } \\
\hline \multicolumn{5}{|c|}{ c. $-856 \mathrm{~A}>\mathrm{G}$} \\
\hline AA (A) & 50 & 45.3 & 675 & 67.6 \\
\hline $\mathrm{AG}$ & 223 & 44.9 & & \\
\hline GG (G) & 226 & 10.0 & 323 & 32.4 \\
\hline \multicolumn{5}{|c|}{ c. $-207 \mathrm{~T}>\mathrm{C}$} \\
\hline $\mathrm{TT}(\mathrm{T})$ & 226 & 45.6 & 676 & 68.1 \\
\hline $\mathrm{TC}$ & 224 & 45.2 & & \\
\hline $\mathrm{CC}(\mathrm{C})$ & 46 & 9.3 & 316 & 31.9 \\
\hline \multicolumn{5}{|c|}{ c. $1714 \mathrm{C}>\mathrm{A}$} \\
\hline $\mathrm{CC}(\mathrm{C})$ & 5 & 1.0 & 101 & 10.2 \\
\hline $\mathrm{CA}$ & 91 & 18.3 & & \\
\hline $\mathrm{AA}(\mathrm{A})$ & 400 & 80.6 & 891 & 89.8 \\
\hline
\end{tabular}

c. $87 \mathrm{~A}>\mathrm{G}$ and IL-12R $\beta 2$ c.- $756 \mathrm{~T}>\mathrm{C}$ ) as determined by chi-square test. Linkage disequilibrium was evaluated for all pairs of SNP using $\mathrm{r}^{2}$. The $\mathrm{r}^{2}$ values ranged from 0.004 to 1.000 . Two of the SNP (IL-12R $\beta 2$ c.$406 \mathrm{~A}>\mathrm{G}$ and IL-12R $\beta 2$ c.-511A $>\mathrm{G})$ were in perfect disequilibrium $\left(\mathrm{r}^{2}=1\right)$, so only SNP IL-12R $\beta 2$ c.-511A $>\mathrm{G}$ was used in the analyses.

Allele Substitution Effect. Although no significant associations were found with SCS, analyses revealed significant associations (comparison-wise $P<0.05$ ) between SNP IL-12Rß2 c.-511A > G and MYLD and PYLD, SNP IL-12R 32 c. $87 \mathrm{~A}>\mathrm{G}$ and MYLD and PYLD, and SNP IL-12R $\beta 2$ c.2957A > C and PYLD. Table 4 presents the estimated allele substitution effects for SNP IL-12R $\beta 2$ c.-511A > G, IL-12R $\beta 2$ c.87A > G, and IL-12R $\beta 2$ c.2957A > C for MYLD and PYLD. For SNP IL-12R $\beta 2$ c.-511A > G, no animals had genotype

Table 4. Significant associations (allele substitution effects \pm SE; $P$-value in parentheses) of SNP in the IL-12 receptor subunit $\beta 2$ (IL12Rß2) gene with MYLD and PYLD in Holsteins

\begin{tabular}{llr}
\hline & \multicolumn{2}{c}{ Trait $^{1}$} \\
\cline { 2 - 3 } SNP & MYLD $(\mathrm{kg})$ & PYLD $(\mathrm{kg})$ \\
\hline c. $-511 \mathrm{~A}>\mathrm{G}$ & $+612.3 \pm 225.6(0.007)^{*}$ & $+13.2 \pm 6.4(0.041)$ \\
c.87A $>\mathrm{G}$ & $-544.3 \pm 207.7(0.010)$ & $-11.7 \pm 5.9(0.049)$ \\
c.2957A $>\mathrm{C}$ & & $-4.7 \pm 2.0(0.021)$ \\
\hline
\end{tabular}

${ }^{1}$ MYLD $=$ milk yield; PYLD $=$ protein yield.

*Significant at a trait-wise $5 \%$ false discovery rate. 
GG. Therefore, only genotypes AA and AG were used to estimate the allele substitution effect. The $G$ allele was associated with higher milk and protein yield drEBV compared with the A allele. For SNP IL-12R $\beta 2$ c.87A $>\mathrm{G}$, the A allele was associated with increased milk and protein yield. Last, for SNP IL-12Rß2 c.2957A > $\mathrm{C}$, the A allele was associated with increased protein yield drEBV. The association of SNP IL-12R $\beta 2$ c.-511A $>\mathrm{G}$ and MYLD also held its significance at a trait-wise false discovery rate of $5 \%$.

The estimated polygenic variance in model (1) explained 76.8 and $79.5 \%$ of the total estimated variance of drEBV (polygenes + residual variance) for protein and milk yield, respectively. The same features when SNP in the IL-12R $\beta 2$ gene were not included in the model were 78.5 and $80.4 \%$, respectively, indicating that the polygenic effects capture part of the variation associated with the SNP in the IL-12R $\beta 2$ gene when the SNP were dropped from model [1].

Haplotype Reconstruction. Two IL-12R $\beta 1$ SNP (c.81T > C, c.1500T > C), 4 IL-12Rß2 SNP (c.2957A $>$ C, c. $87 \mathrm{~A}>\mathrm{G}$, c. $-511 \mathrm{~A}>\mathrm{G}$, c. $-756 \mathrm{~T}>\mathrm{C}$ ), and 3 IL-23R SNP (c.-856A > G, c.-207T > C, c.1714C > A) were used for haplotype reconstruction. The estimated frequencies of all haplotypes are in Table 5. Haplotypes (Hap) for each gene were reconstructed separately. The most common IL-12R $\beta 1$ haplotype among the Holstein bulls was TT (Hap1) with an estimated frequency of 0.54. The most common IL-12R $\beta 2$ haplotype among Holstein bulls was AGAT (Hap1) with an estimated frequency of 0.43 . More than $57 \%$ of the IL-12R 32 haplotypes observed were either AGAT (Hap1) or CGAT (Hap4). The rest of the 12 haplotypes had a combined frequency of about $43 \%$. Five IL-12R $\beta 2$ haplotypes, Hap10 to Hap14, were very rare in the population, with a combined frequency of $4.3 \%$. The most common IL-23R haplotype was ATA (Hap3) with an estimated frequency of 0.54 . More than $74 \%$ of the IL-23R haplotypes present were either ATA (Hap3) or GCA (Hap4). Two IL-23R haplotypes, Hap5 and Hap6, were rare in the population, with a combined frequency of $1.5 \%$.

Haplotype Analyses. Because IL-12R $\beta 2$ haplotypes $10,11,12,13$, and 14 together represented only $4.3 \%$ of all haplotypes, they were pooled (HapP) as a single effect for further analyses. Similarly, because IL-23R haplotypes 5 and 6 together represented less than $2 \%$ of all haplotypes, they were pooled (HapP) as a single effect for further analyses. The effect of the most common haplotype for each gene (IL-12R $\beta 1$ Hap1; IL-12Rß2 Hap1; IL-23R Hap3) was used as a control, and the rest of the haplotypes were contrasted against this haplotype. Analyses revealed significant differences with respect to the most frequent haplotype (comparison-wise $P<0.05$ ) for IL-12R $\beta 2$ Hap3 and HapP for MYLD and Hap4 for PYLD and IL-23R Hap4 and Hap8 for LACTP (Table 6).

As for the allele substitution model, the polygenic effects captured part of the variance associated with the haplotypes in the IL-12R $\beta 2$ gene when the haplotypes were not fit in the model. The estimated polygenic variance explained 76.0 and $78.3 \%$ of the total estimated variance of drEBV (polygenes + residual variance) for protein and milk yield, respectively, when haplotypes were fit in the model. The same features, when haplotypes for SNP in the IL-12R $\beta 2$ gene were dropped from the model, were 77.8 and $79.8 \%$, respectively.

\section{DISCUSSION}

Interleukin-12 and IL-23 play an important role in regulating the inflammatory and immune response, and we hypothesized that variants in the genes coding the receptors for these cytokines contributed to individual variation in health-related and production traits in dairy cattle. Therefore, the main objective of this study was to identify SNP in the bovine IL-12R $\beta 1$, IL-12R $\beta 2$, and IL-23R genes. The SNP were then used to test for associations between these SNP health-related and production traits.

Because exons and promoter regions were targeted for SNP detection, and 40 animals were used to create the DNA pool for SNP detection, it is unlikely that all SNP in the population were identified. Single nucleotide polymorphisms $(\mathrm{n}=2)$ were identified in the IL-12R $\beta 1$ gene; both SNP (c.81T > C, c.1500T > C) were synonymous and located in the coding region of the gene. Five SNP were identified in the IL-12R $\beta 2$ gene, 1 of which was a synonymous SNP found in exon 2 (c.87A $>$ G) of this gene. The SNP c.2957A $>$ C is located in the $3^{\prime}$ UTR of the gene. Three SNP were identified in the $5^{\prime}$ upstream region (c.-756T $>$ C, c. $-511 \mathrm{~A}>\mathrm{G}$, c. $-406 \mathrm{~A}>\mathrm{G})$ in the putative promoter of the gene; SNP (c.-406A > G, c.-511A > G) were found to be located within potential transcription-factor binding sites (STAT6, NR2F, CLOX, DEAF1, and POZ binding sites). Although little work has been done on many of these transcription factors, there is evidence that STAT6 may play an important role in the regulation of this gene. Jin et al. (2008) found that STAT6-deficient mice have a decreased ability to produce IFN- $\gamma$ in response to infection with Toxoplasma gondii, as well as decreased production of IL-12 p40. Additionally, it has been suggested that alterations in the STAT6 pathway may play a crucial role in the pathogenesis of human patients with Crohn's disease (Klein et al., 2005).

Although some of the SNP in the IL-12R $\beta 2$ gene appear to be biologically meaningful, the present study found no association between these SNP and SCS. As- 
Table 5. Haplotype reconstruction for SNP in IL-12 receptor subunit $\beta 1$ (IL12R $\beta 1$ ), IL-12 receptor subunit $\beta 2$ (IL12R $\beta 2$ ), and IL-23 receptor (IL23R) genes and their associated frequencies in the Holstein bulls

\begin{tabular}{|c|c|c|c|c|c|}
\hline \multirow[b]{2}{*}{ Haplotype } & \multicolumn{2}{|c|}{ SNP (IL12R $\beta 1)$} & & & \multirow[b]{2}{*}{ Frequency } \\
\hline & c. $81 \mathrm{~T}>\mathrm{C}$ & c. $1500 \mathrm{~T}>\mathrm{C}$ & & & \\
\hline Нар1 & $\mathrm{T}$ & $\mathrm{T}$ & & & 0.538 \\
\hline Hap2 & $\mathrm{C}$ & $\mathrm{C}$ & & & 0.212 \\
\hline Нар3 & $\mathrm{C}$ & $\mathrm{T}$ & & & 0.177 \\
\hline \multirow[t]{3}{*}{ Hap4 } & $\mathrm{T}$ & $\mathrm{C}$ & & & 0.074 \\
\hline & \multicolumn{4}{|c|}{ SNP (IL12Rß2) } & \\
\hline & c. $2957 \mathrm{~A}>\mathrm{C}$ & c. $87 \mathrm{~A}>\mathrm{G}$ & c. $-511 \mathrm{~A}>\mathrm{G}$ & c. $-756 \mathrm{~T}>\mathrm{C}$ & \\
\hline Hap1 & $\mathrm{A}$ & G & $\mathrm{A}$ & $\mathrm{T}$ & 0.433 \\
\hline Hap2 & $\mathrm{C}$ & $\mathrm{G}$ & $\mathrm{A}$ & $\mathrm{C}$ & 0.046 \\
\hline Hap3 & A & $\mathrm{G}$ & $\mathrm{A}$ & $\mathrm{C}$ & 0.072 \\
\hline Hap4 & $\mathrm{C}$ & $\mathrm{G}$ & $\mathrm{A}$ & $\mathrm{T}$ & 0.142 \\
\hline Нар5 & $\mathrm{C}$ & A & A & $\mathrm{C}$ & 0.081 \\
\hline Нар6 & $\mathrm{C}$ & A & A & $\mathrm{T}$ & 0.029 \\
\hline Hap7 & A & A & A & $\mathrm{T}$ & 0.041 \\
\hline Нар8 & A & A & A & $\mathrm{C}$ & 0.084 \\
\hline Hap9 & A & A & $\mathrm{G}$ & $\mathrm{T}$ & 0.028 \\
\hline Hap10 & A & A & G & $\mathrm{C}$ & 0.012 \\
\hline Hap11 & $\mathrm{C}$ & A & G & $\mathrm{T}$ & 0.015 \\
\hline Hap12 & $\mathrm{C}$ & A & G & $\mathrm{C}$ & 0.006 \\
\hline Hap13 & $\mathrm{C}$ & $\mathrm{G}$ & $\mathrm{G}$ & $\mathrm{T}$ & 0.007 \\
\hline \multirow[t]{3}{*}{ Hap14 } & A & $\mathrm{G}$ & $\mathrm{G}$ & $\mathrm{T}$ & 0.003 \\
\hline & \multicolumn{3}{|c|}{ SNP (IL23R) } & & \\
\hline & c. $-856 \mathrm{~A}>\mathrm{G}$ & c. $-207 \mathrm{~T}>\mathrm{C}$ & c. $1714 \mathrm{C}>\mathrm{A}$ & & \\
\hline Hap1 & $\mathrm{A}$ & $\mathrm{C}$ & $\mathrm{A}$ & & 0.076 \\
\hline Hap2 & G & $\mathrm{T}$ & $\mathrm{A}$ & & 0.079 \\
\hline Hap3 & $\mathrm{A}$ & $\mathrm{T}$ & $\mathrm{A}$ & & 0.537 \\
\hline Нар4 & G & $\mathrm{C}$ & A & & 0.206 \\
\hline Hap5 & $\mathrm{G}$ & $\mathrm{T}$ & $\mathrm{C}$ & & 0.008 \\
\hline Hap6 & $\mathrm{A}$ & $\mathrm{C}$ & $\mathrm{C}$ & & 0.007 \\
\hline Нар7 & G & $\mathrm{C}$ & $\mathrm{C}$ & & 0.034 \\
\hline Hap8 & A & $\mathrm{T}$ & $\mathrm{C}$ & & 0.053 \\
\hline
\end{tabular}

sociations were, however, observed between IL-12R $\beta 2$ SNP (c.-511A > G, c.87A > G, c.2957T > A) and milk and protein yields. Such associations may be a result of linkage between these SNP and other genes on the same chromosome that have a significant effect on these production traits. Significant QTL, for example, have been found on BTA3 for all 3 production traits used in the analyses. The confidence interval for $1 \mathrm{QTL}$ for milk yield, for example, overlaps the position of the IL-12R $\beta 2$ gene, 77,660 kbp (Ashwell et al., 2004).

Table 6. Significant association (deviation $\pm \mathrm{SE} ; P$-value ${ }^{1}$ in parentheses) from the most frequent haplotypes of SNP haplotypes for IL-12 receptor subunit $\beta 2$ (IL12R 32 ) and IL-23 receptor (IL23R) genes with deregressed EBV for MYLD, PYLD, and LACTP

\begin{tabular}{lccc}
\hline & \multicolumn{3}{c}{ Trait $^{2}$} \\
\cline { 2 - 4 } Haplotype & MYLD (kg) & PYLD (kg) & LACTP (score) \\
\hline IL12R32 & & \\
Hap3 (AGAC) & $-815.2 \pm 381.0(0.034)$ & & \\
Hap4 (CGAT) & $495.0 \pm 225.7(0.029)$ & & \\
HapP & & & \\
IL23R & & & $1.2 \pm 0.6(0.037)$ \\
Hap4 (GCA) & & $2.4 \pm 0.9(0.009)$ \\
Hap8 (ATC)
\end{tabular}

${ }^{1}$ Probability of $t$-test.

${ }^{2} \mathrm{MYLD}=$ milk yield PYLD $=$ protein yield LACTP $=$ lactation persistency.

${ }^{3} \mathrm{HapP}=$ pooled haplotypes 10 to 14 . 
Three SNP were also identified in the IL-23R gene, including a nonsynonymous SNP found in exon 11 (c.1714C > A, with the $\mathrm{C}$ allele coding for proline and A allele coding for threonine). Alignment of the bovine IL-23R sequence using the software Uniprot (http:// www.uniprot.org) with the human IL-23R sequence suggests that this SNP is located in the cytoplasmic domain of the receptor when the final protein is formed. A SNP within this domain may affect the internal signaling of the receptor within the cell after binding to IL-23. For example, the chemical structure of threonine and proline are very different, with threonine containing a hydroxy side chain and proline being a cyclic amino acid. The other $2 \mathrm{SNP}$ were identified in the $5^{\prime}$ upstream region (c.-856A $>$ G, c.-207T > C) in the putative promoter of the gene. Both are located within a single potential transcription factor binding site (Figure 3 ). Although the significance of these SNP is biologically relevant, in that they may affect gene expression or function, the present study found no associations between the analyzed IL-23R SNP and the investigated traits. Haplotype reconstruction of the IL-12R $\beta 2$ SNP revealed 14 of the 16 possible haplotypes to be likely segregating in the sampled bulls. The reason for seeing only 14 haplotypes may be a result of intense selection within the Holstein population. No differences between the linear effects of the haplotypes on SCS were observed. However, associations between IL-12R $\beta 2$ Hap3 and milk yield and IL-12R $\beta 2$ Hap4 and protein yield were observed; Hap3 and Hap4 were associated with decreased milk and protein production, respectively. These associations with production traits could be a result of the effect of the QTL identified on BTA3 for milk, fat, and protein yields. The IL-23R Hap4 and Hap8 were associated with increased LACTP. The fact that an association is seen with these haplotypes in IL23R, but not with the individual SNP, could be a result of allele interaction within the same chromosome, with the combination of alleles having an effect. Haplotype reconstruction of the IL-12R $\beta 1$ SNP revealed 4 haplotypes, but no significant associations were observed.

\section{CONCLUSIONS}

Two SNP in IL-12R $\beta 1,5$ SNP in IL-12R $\beta 2$, and 3 $\mathrm{SNP}$ in IL-23R were discovered in a sample of $492 \mathrm{Ca}-$ nadian Holstein bulls. Four SNP (IL-12R $\beta 2$ c.-511A > G, IL-12R 32 c.-406A > G, IL-23R c.-856A > G, IL-23R c.-207T > C) were located within potential transcription factor binding sites, and SNP IL-23R c.1714C $>$ A was nonsynonymous, potentially located in the cytoplasmic receptor domain. Because of the potential of these SNP to alter gene expression and protein function, they warrant future investigation. Although no association between these SNP and SCS was established in this study, associations were found between 3 of these SNP (IL-12R $\beta 2$ c. $-511 \mathrm{~A}>\mathrm{G}$, IL-12R $\beta 2$ c.87A $>$ G, IL-12R 32 c.2957T > A, ) and 2 haplotypes in the IL-12R $\beta 2$ gene with drEBV for milk and protein yields. Additional analysis of the haplotypes also revealed an association of 2 haplotypes in IL-23R gene with drEBV for LACTP. These associations will require further investigation with respect to their biological and practical relevance before they can be considered as genetic markers for selection purposes.

\section{REFERENCES}

Alain, K., N. A. Karrow, C. Thibault, J. St-Pierre, M. Lessard, and N. Bissonnette. 2009. Osteopontin: An early innate immune marker of Escherichia coli mastitis harbors genetic polymorphisms with possible links with resistance to mastitis. BMC Genomics 10:444.

Alluwaimi, A. M., C. Leutenegger, T. Farver, P. Rossitto, W. Smith, and J. Cullor. 2003. The cytokine markers in Staphylococcus aureus mastitis of bovine mammary gland. J. Vet. Med. B Infect. Dis. Vet. Public Health 50:105-111.

Ashwell, M. S., D. W. Heyen, T. S. Sonstegard, C. P. Van Tassell, Y. Da, P. M. Van Raden, M. Ron, J. I. Weller, and H. A. Lewin. 2004. Detection of quantitative trait loci affecting milk production, health and reproduction traits in Holstein cattle. J. Dairy Sci. $87: 468-475$.

Boettcher, P. J., G. Pagnacco, and A. Stella. 2004. A Monte Carlo approach for estimation of haplotype probabilities in half-sib families. J. Dairy Sci. 87:4303-4310.

Freidin, M., A. Rudko, O. Kolokolova, A. Strelis, and V. Puzyrev. 2006. Associations between the $1188 \mathrm{~A} / \mathrm{C}$ polymorphism in the human IL12B gene and Th1-mediated infectious diseases. Int. J. Immunol. 33:231-232.

Gilmour, A. R., B. R. Cullis, S. J. Welham, and R. Thompson. 2000. ASREML Reference Manual. IACR-Rothamsted Experimental Station, Harpenden, UK.

Henderson, C. R. 1988. Use of an average numerator relationship matrix for multiple-sire joining. J. Anim. Sci. 66:1614-1621.

Hill, W. G., and A. Robertson. 1968. Linkage disequilibrium in finite populations. Theor. Appl. Genet. 38:226-231.

Jin, D., M. Takamoto, T. Hu, S. Taki, and K. Sugane. 2008. STAT6 signaling is important in CD8+ T-cell activation and defense against Toxoplasma gondii infection in the brain. Immunology 125:1-9.

Klein, W., A. Tromm, C. Folwaczny, M. Hagedorn, N. Duerig, J. Epplen, W. Schmiegel, and T. Griga. 2005. The G2964A polymorphism of the STAT6 gene in inflammatory bowel disease. Dig. Liver Dis. 37:159-161.

Langrish, C., B. McKenzie, N. Wilson, R. Malefyt, R. Kastelein, and D. Cua. 2004. IL-12 and IL-23: Master regulators of innate and adaptive immunity. Immunol. Rev. 202:96-105.

Leyva-Baca, I., F. Schenkel, J. Martin, and N. Karrow. 2008. Polymorphisms in the $5^{\prime}$ upstream region of the CXCR 1 chemokine receptor gene, and their associations with somatic cell score in Holstein cattle in Canada. J. Dairy Sci. 91:407-417.

Leyva-Baca, I., F. Schenkel, B. S. Sharma, G. B. Jansen, and N. A. Karrow. 2007. Identification of single nucleotide polymorphisms in the bovine CCL2, IL8, CCR2 and IL8RA genes and their association with health and production in Canadian Holsteins. Anim. Genet. 38:198-202.

Pant, S., F. Schenkel, I. Leyva-Baca, B. Sharma, and N. Karrow. 2007. Identification of single nucleotide polymorphisms in bovine CARD15 and their associations with health and production traits in Canadian Holsteins. BMC Genomics 8:421.

Rupp, R., and D. Boichard. 2003. Genetics of resistance to mastitis in dairy cattle. Vet. Res. 34:671-688. 
Sharma, B., I. Leyva, F. Schenkel, and N. Karrow. 2006. Association of toll-like receptor 4 polymorphisms with somatic cell score and lactation persistency in Holstein bulls. J. Dairy Sci. 89:3626-3635.

Shook, G. E., and M. Schutz. 1994. Selection on somatic cell score to improve resistance to mastitis in the United States. J. Dairy Sci. $77: 648-658$.

Tost, J., and I. Gut. 2005. Genotyping single nucleotide polymorphisms by MALDI mass spectrometry in clinical applications. Clin. Biochem. 38:335-350.

van de Vosse, E., E. Lichtenauer-Kaligis, J. Dissel, and T. Ottenhoff. 2003. Genetic variations in the interleukin-12/interleukin-23 re- ceptor ( $\beta 1)$ chain, and implications for IL-12 and IL-23 receptor structure and function. Immunogenetics 54:817-829.

Winfrey, M. R., M. A. Rott, and A. T. Wortman. 1997. Unraveling DNA: Molecular Biology for the Laboratory. Prentice Hall, Upper Saddle River, NJ.

Yilmaz, V., S. Yentur, and G. Saruhan-Direskeneli. 2005. IL-12 and IL-10 polymorphisms and their effects on cytokine production. Cytokine 30:188-194.

Zeng, Z. B., T. Wang, and W. Zou. 2005. Modeling quantitative trait loci and interpretation of models. Genetics 169:1711-1725. 